\title{
THE STRUCTURE AND PROPERTIES OF MOLECULAR TREES AND NETWORKS
}

\author{
MANFRED GORDON and S. B. ROSS-MURPHY
}

Institute of Polymer Science, University of Essex, Colchester, UK

\begin{abstract}
The gel point is a well-understood critical point in polymer science (Flory, Stockmayer). A summary is given of the resolution of those aspects which may seem paradoxical at first sight. The relevant equations for the basic paradigm of $f$-functional polycondensation are very simple. Special attention is paid to the critically branched state of materials not far from the gel point. The quasiinvariance principle is explained according to which all solution properties appear ultimately to level off as the critical conversion is approached from below. The 'Malthusian' packing paradox is resolved by a proper treatment of the ring-chain competition situation, which also disposes of the spurious divergence of the rate of cyclization predicted by a more naive theory.

Network theories not based on extinction probability (Charlesby) are not worth considering. The proper definition of an elastically active network chain (EANC) was based on this concept by Scanlan and by Case in 1960, and it greatly simplifies the graph-like-state theories of network structure (usually called 'network topology'). It allows classical rubber elasticity theory to be applied near the gel point. The point is characterised by a fifth-order Ehrenfest transition due to the contribution of long-range correlations to the configurational free energy. Though directly relevant data on reversible gelation are not available, data on isothermal crosslinking of very diverse systems support this analysis. Except possibly for some highly crosslinked systems, the parameter $\bar{M}_{\mathrm{c}}$ (mean chain length between 'crosslinks') is an undesirable ingredient of elasticity or swelling theories. Chain-end corrections are quite undesirable (and usually done incorrectly). Everything is correctly and more simply formulated in terms of Scanlan-Case EANCs.

The proper understanding of the gel point as a critical point allows the construction of reduced plots, illustrated with temperature superposition of experimental modulus-conversion plots (by $\mathbf{M}$. Judd) for aqueous gelatin jellies. These fit reasonably to the basic model involving triple-helix junction zones, essentially without adjusting arbitrary parameters. Such reducedvariable treatments for critically branched materials eliminate the otherwise inescapable difficulties of characterization, purity, electrolyte content, etc., from gelatin research.
\end{abstract}

\section{INTRODUCTION}

Chemical physics derives most of its interest from the existence of critical points. One of the very few that is fully intelligible theoretically, largely thanks to the analysis of Flory ${ }^{1}$ and Stockmayer ${ }^{2}$. is also the simplest and the most important, because no living being exists on Earth whose material 
substance has not passed through this critical point. I mean, of course, the gel point.

Compared with the critical point of three-dimensional Ising models (e.g. the Curie point), gelation gives rise to only paltry paradoxes. I apologize for using this lecture to explain them once again before I turn to recent developments.

The mathematical simplicity of gelation theory. like that of the onedimensional Ising model for the helix-coil transition ${ }^{3}$, stems from the onedimensional ('graph-like' or floppy) nature of the particles in the model. From the start. the theory was sufficiently general to embrace all kinds of gelling materials within one framework: the conversion parameter $\alpha$ of functionalities, and the valency or 'functionality' $f$ of a repeat unit, are the sole parameters of the basic theory, equally defined for polycondensates. polyfunctional vinyls or vulcanizates made by crosslinking primary chains. Later, further intermediate parameters were introduced, but they are calculated directly from $\alpha$ and $f$. Especially, Charlesby ${ }^{4}$ re-invented the general notion of the extinction probability $v$ for polymer science (it was already known in the theory of branching processes ${ }^{5}$ ), while Scanlan ${ }^{6}$ and Case ${ }^{7}$ defined the number $N_{\mathrm{e}}$ of elastically effective network chains (EANCs) in a perfectly generalizable way. Further generalization of the basic parameters $\alpha$ and $f$ to multicomponent (vectorial) form $\alpha_{1}, \alpha_{2}, \ldots$ and $f_{1}, f_{2}, \ldots$ to deal with more complex copolymeric systems or primary chain distributions is largely a routine matter. The generality of the first-approximation theory of crosslinking and networks will allow us to illustrate calculation of all interesting and paradoxical aspects on the basis of the simplest model situation (Section 2). The resulting almost trivial formulae are usually verified experimentally with high precision. Although chemists accept the point-atoms of the ideal-gas model, they are revolted by the floppy molecules of the graph-like state. However, on the ideal-gas model, the difference in standard entropy at $25^{\circ} \mathrm{C}$ between n-octane and 2,2,3,3-tetramethyl butane is zero, while on the graphlike floppy model ${ }^{8}$ it is $8 R \ln 3=55.2 \mathrm{~J} \mathrm{~K}^{-1} \mathrm{~mol}^{-1}$. The experimental value is 74.3 , so that there the graph-like state forms a typically reasonable first approximation.

\section{THE BASIC PARADIGM OF CHEMISTRY AND THE GEL POINT}

Chemistry is the science of the making and breaking of bonds. The simplest starting model (Figure 1) is, therefore, constructed as follows : a large number of identical one-dimensional particles are enclosed in a box, each with a number $f$ of functionalities or binding sites, which are identical, preferably by virtue of molecular symmetry. These are allowed to make and break bonds and reach equilibrium under the simplest thermodynamic regime, viz. bondadditivity of the free enthalpy. The reaction $\mathrm{H}+\mathrm{H}=\mathrm{H}_{2}$ is a degenerate case $(f=1)$ of this basic paradigm of chemistry, first formulated in 1941 by Flory ${ }^{1}$ under the name of $f$-functional random polycondensation; but few British chemists have yet heard of it. Without further assumptions, this model leads to a critical point. This is shown in Figure 2 for the case $f=3$. e.g. the etherification of 1,3,5-trimethylol benzene ${ }^{9}$-in terms of plots of the mean 

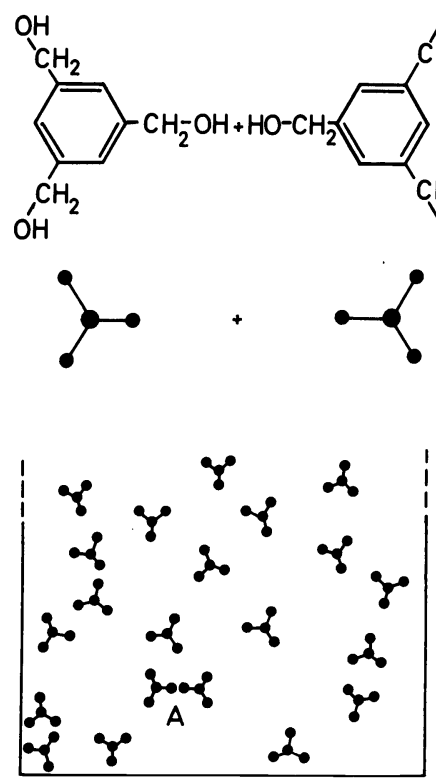

(b)
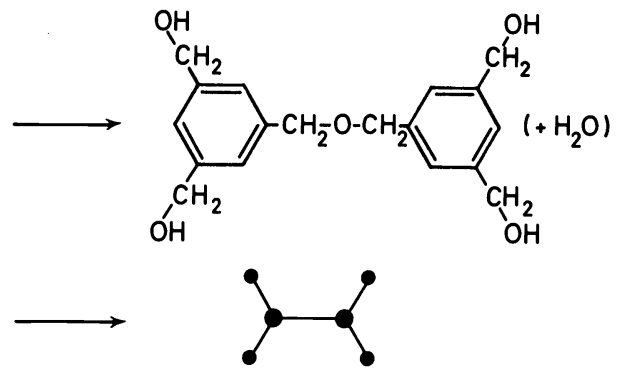

(a)

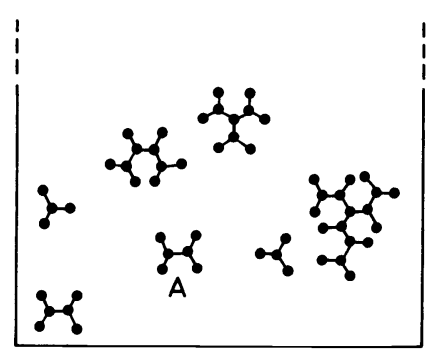

(c)

Figure 1. Graph-like state model for the random trifunctional condensation of 1,3,5-trimethylol benzene. Top, formation of dimer in chemical notation. (a), Reduction to civilized (graph-like) state, which contains the relevant information. (b) and (c), Basic paradigm of the graph-like particle in a box, respectively at conversions $\alpha=0$ and $\alpha>0$. A, formation of dimer. The box (c) also shows the two possible isomeric tetramers at the top

sizes $x_{\max , 1}$ and $x_{\max , 2}$ of the largest ${ }^{10}$ and second-largest molecules as functions of relative conversion $\alpha / \alpha_{c}$, where $\alpha_{c}$ is the critical conversion. In principle, the equilibrium conversion $\alpha$ can be adjusted by varying the pressure or temperature. In practice, the mixture $\mathrm{P}_{2} \mathrm{O}_{5} / \mathrm{POCl}_{3}$ can be treated as an example of the model, and here $\alpha$ is controlled by the stoichiometric ratio ${ }^{11}$ (cf. Groenweghe, Payne and Van Wazer ${ }^{12}$ ). Also irreversible but random (ring-free) condensations pass through states (distributions) indistinguishable from those of the reversible equilibrium model $\left(\right.$ Stockmayer $^{2}$ ). The quantities $x_{\max , 1}$ and $x_{\max , 2}$ fluctuate at fixed $\alpha / \alpha_{\mathrm{c}}$ from one test-tube sample to another but we have plotted the mean, denoted by a bar, over many test-tubes. We see how, over a very narrow range of conversion, the largest molecule increases on average by many powers of ten, while the second-largest follows close behind up to the critical point, and then quickly declines. The critical phenomenon is easy to explain qualitatively. An $x$-mer molecule (cf. Figure 1) must have $x-1$ inter-unit links to hold the $x$ units together, and $f x-2 x+2$ terminals ('free functionalities'). This follows for all isomers from elementary combinatorial graph theory. Thus a graph-like $x$-mer species in the box has degradation rate $\propto(x-1)$ and a growth rate $\propto f x-2 x+2$. Accordingly. when $f>2$, the chance of further growth, rather than degradation, as the equilibrium is driven towards higher conversions $\alpha$, is the more favourable 


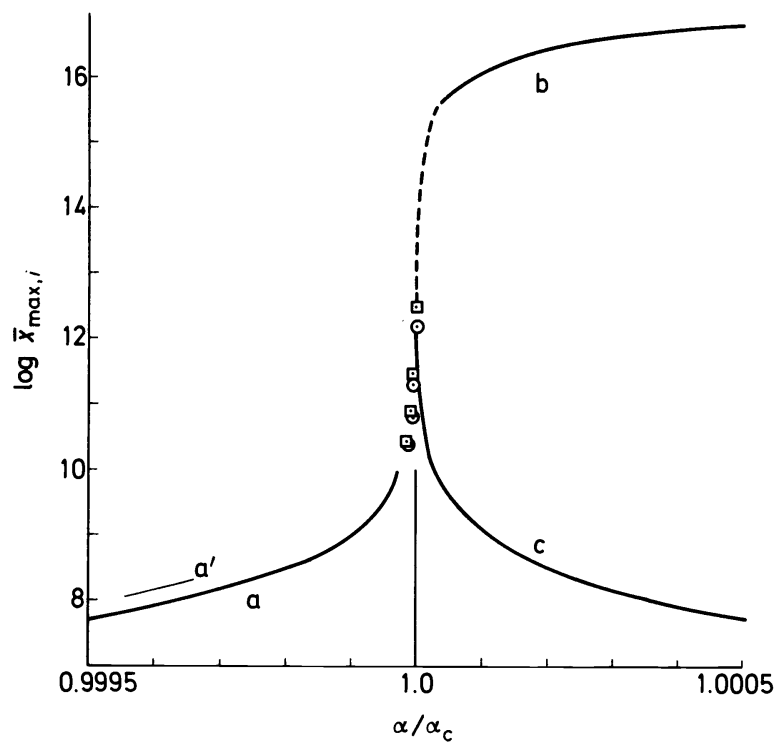

Figure 2. Plots of the logarithms of the mean DPs of the two biggest molecules against relative conversion near the gel point. The mean is taken over a large set of systems of a few mg of trifunctional polycondensates, each with $2 \times 10^{19}$ repeat units. Curve a, calculated from equations (A1) and (A3) (with the asymptotic distribution, equation 2), is the lower limit, and a' the upper limit, arising from statistical approximations (equation $A 7)$; both refer to the largest $\left(\left(\bar{x}_{\max }, 1\right)\right.$ and second-largest $\left(\bar{x}_{\max , 2}\right)$ molecule. These become separately distinguishable very close to the gel point $\left(\alpha / \alpha_{c}=1\right)$ as $\square \bar{x}_{\max , 1}, O \bar{x}_{\max , 2}$. At the gel point the exact points shown differ by $\frac{2}{3} \log 3$. Curve $\mathrm{b}$ represents $\bar{x}_{\max , 1}$, now the gel molecule, calculated as $\bar{x}_{\max , 1}=\bar{x}_{\text {gel }}=2 \times 10^{19}$ $\left\{1-\left(\alpha^{-1}-1\right)^{3}\right\}$. Curve c represents $\bar{x}_{\max , 2}$, the largest molecule in the sol fraction, found by reflection of a (and of the circles) in the line $\alpha / \alpha_{c}=1$

the larger $x$, i.e. the larger the species. As a result, one single species, over a quite narrow range of conversion, finally outgrows all others in size, which explains Figure 2.

The details for calculating $\bar{x}_{\max , i}$ are given in appendix A. Here we note merely the basic equations underlying the criticality. Stockmayer gave the weight distribution $w_{x}$ of $x$-meric species in the $f$-functional random polycondensate model :

$$
w_{x}=\frac{(f x-x) ! f}{(f x-2 x+2) !(x-1) !} \alpha^{x-1}(1-\alpha)^{f x-2 x+2}
$$

and his formula for the asymptotic form $\left(\alpha \rightarrow \alpha_{c}\right)$ may be written :

$$
w_{x}=\left(B A^{x} / x^{\frac{3}{2}}\right)+o(x) \quad(f>2)
$$

where

$$
A=(1-\alpha)^{f-2} \alpha(f-1)^{f-1} /(f-2)^{f-2}
$$

and

$$
B=(1-\alpha)^{2} f(f-1)^{\frac{1}{2}} /(2 \pi)^{\frac{1}{2}} \alpha(f-2)^{5 / 2}
$$


In particular, at the gel point itself $\left(\alpha=\alpha_{d}\right)$ we find the asymptotic distribution (for $x>c a$. 100), thus

$$
\left.w_{x}=\text { const. } x^{-\frac{3}{2}} \quad \text { (const. } \sim 1\right)
$$

independently of $f$ if $f>2$.

Figure 2 solves the conceptual difficulty some beginners experience when they encounter the term 'infinite' molecule instead of $\bar{x}_{\max }\left(\equiv x_{\max , 1}\right)$. When the size of the system increases by a factor $c$, say. $\bar{x}_{\max , \text { crit }}$ increases by a factor $c^{\frac{2}{3}}$ (equation A1), and tends to infinity with $c$.

We revert briefly to the notion of compositional variations (at fixed $\alpha$ ) from sample to sample. Figure 3 shows schematically how the smooth asymptotic

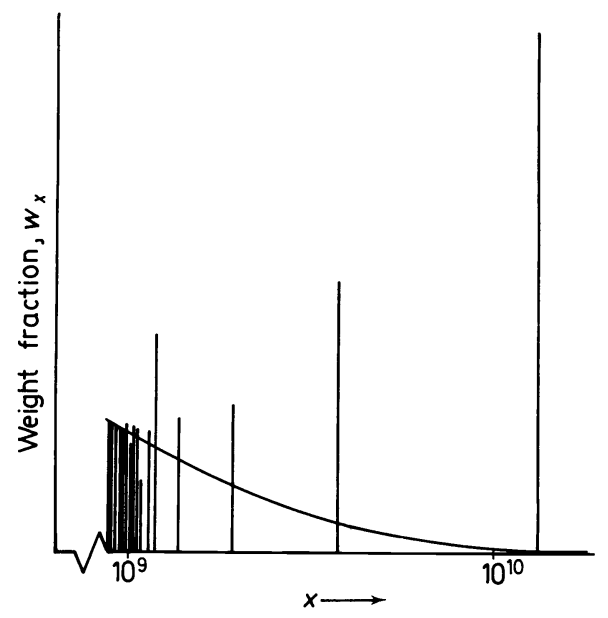

Figure 3. Statistical effect of finite number of repeat units on the tail of the weight fraction distribution near the gel point (schematic). At low molecular weights the smooth theoretical curve for an infinite system is an excellent representation. At high molecular weights each single $x$-mer molecule actually present exceeds its weight-fraction quota, and the spacing between the sizes of representative $x$-mers actually realized increases. The last molecule realized on the right is $\bar{x}_{\max , 1}$

distribution 2 must 'fade out' in practice at high enough $x$ to hand over to the discrete distribution of isolated large molecules, ever more widely spaced out, in a statistical way, until we reach $x_{\max , 1}$. Fluctuations from sample to sample are most obvious in this high molecular weight region. But they exist even at low molecular weight. We may calculate from Pólya's formula ${ }^{13}$ that there are approximately $10^{40}$ different isomers in the 100 -mer fraction of tetrafunctional polycondensate of the type under discussion. Only an infinitesimal fraction of these can be present in a given sample, and no two samples are ever going to be identical in practice. But the physical properties will generally be indistinguishable from sample to sample, because a representative selection of species generally arises on a statistical basis (except possibly extremely close to the gel point). To deal with equilibrium fluctuations more precisely if it becomes necessary, Whittle ${ }^{14}$ showed how the kinetic equations 
for $f$-functional random polycondensation could be elegantly generalized to a stochastic form, from which, in accord with the classical Gibbs distribution, the number fraction $n_{x}$ emerges as a Poisson variable at fixed conversion.

\section{QUASI-INVARIANCE PRINCIPLE FOR CRITICALLY BRANCHED SAMPLES ${ }^{10}$}

The quasi-invariance principle for critically branched materials was deduced for $f$-functional random polycondensates ${ }^{10}$. but is no doubt a quite general statistical phenomenon. The principle concerns effects arising from cutting-off of the tail of the molecular weight distribution, for convenience taken to occur at a sharp limiting value, $x_{\text {lim }}$, say. Three reasons for such curtailment are important in practice :

(1) The statistical tail-cutting. arising from finiteness of samples (cf. Figures 2 and 3). Species beyond $x_{\max }$, present in the theoretical distribution for infinite samples, are not present in the finite sample, and thus connot contribute towards measurable properties: we may put $\bar{x}_{\text {max }} \sim x_{\text {lim }}$.

(2) Preparative cut-off: species beyond a certain $x_{\text {lim }}$ may be lost. e.g. in removing dust by centrifugation prior to light-scattering.

(3) Instrumental cut-off, e.g. GPC does not respond to molecules beyond a certain $x_{\max }$, depending on the pore size of the gel.

Although in principle present in all polydisperse samples. these three tailcutting effects are most serious in critically branched samples. because of their pathological polydispersity $\left(D P_{w} / D P_{n} \sim 10^{3}\right.$ is easy to attain). The quasi-invariance principle states: if samples ever closer to the gel point are prepared, all physical properties which represent averages over the distribution will rather suddenly level off at a fixed value depending on $x_{\text {lim }}$ the cut-off point of the distribution. In particular, $D P_{w} . D P_{z}$. etc.. instead of appearing to diverge as they should in absence of tail-cutting, will level in this way. The explanation is simple : over the very small range of conversion which is significant (cf. abscissae in Figure 3 ), the values of $w_{x}$ are practically invariant. except for very large $x$. In other words, the gelation phenomenon is concerned almost exclusively with what happens to the few molecules in the very high molecular weight tail (cf. Figure 3). If these molecules are cut away, no effect of further pre-gel conversion will be seen. Some quantitative calculations and plots for the quasi-constancy of the measured $D P_{w}$ were given previously ${ }^{10}$. The onset of a quasi-invariant $\mathrm{DP}_{z}$ is even more sharply accentuated.

The value of the quasi-invariance principle in cell regulation may be exemplified speculatively as follows. Suppose that a cut-off mechanism exists by way of precipitation from solution of molecules exceeding a certain molecular weight threshold. Such a (very sharp) threshold normally arises in the vicinity of a theta temperature. The displacement of the linking equilibrium in solution (by $\mathrm{pH}$, allostery, etc.) towards higher conversion will then be accompanied by precipitation of substantially all newly formed molecules exceeding the threshold size. The quasi-invariance principle ensures that the molecular weight averages and, hence, the viscosity, etc., of the remaining 
liquid phase remains practically unchanged, which might be vital to the continuance of other processes. This kind of mechanism could, accordingly. be of use in the laying down of gel coats or membranes from the cell medium. or in the regulation of membrane permeability ${ }^{15}$. while maintaining constant physical properties in the medium itself.

Flory ${ }^{16}$ also drew attention to the possible importance of tail-cutting effects of the statistical kind (Figure 3) in emulsion systems because of the small mass of the droplets. Indeed. the statistical cut-off point varies as (mass) $)^{\frac{2}{3}}$.

\section{RESOLUTION OF THREE PARADOXES ABOUT GEL MOLECULES}

\subsection{Non-Markovian statistics of gel}

The first paradox about gels arises from the statistics of their one-dimensional structure (molecular graph). It deals with correlations of unlimited range inside any such structure. which are unconnected with the embedding of the graph in (or the existence of) three-dimensional space.

At the gel point the population of components ('molecules') of the graph which represents the chemical sample (i.e. atoms = points; bonds $=$ lines) becomes statistically heterogeneous (Whittle ${ }^{17}$ ). This is illustrated very graphically in Figure 2: the biggest molecule (gel) forms one 'population'; the rest of the sample. led by the relatively tiny second-largest molecule. forms a second population. Of course, the over-all conversion $\alpha$ is continuous through the gel point at $\alpha=\alpha_{\mathrm{c}}$. But the 'private' $\alpha$-value $\left(\alpha_{\mathrm{gel}}\right)$ of the largest molecule rises to a value exceedingly close to $2 / f>1 /(f-1)=\alpha_{c}$, where $f>2$. Here $\alpha=2 / f$ is the exact value of the conversion needed to link $f$ functional units into any shape of infinite tree. On average, two out of the $f$ functionalities of the units in such a tree must be converted to links. This is quite obvious if the shape chosen is a linear chain extending to infinity; but rearranging the links to form a single infinite branched tree does not involve a change in the ratio $\alpha$ of free functionalities to links. The statistical heterogeneity, illustrated by the finite difference between $\alpha_{\text {gel }}$ and $\alpha_{\text {sol }}$, has led some workers to introduce unnecessary complications into the calculation of statistical parameters. The cascade formalism allows the calculation of all statistical parameters for the sol by a routine transformation of the basic link probability generating function (LPGF, from which all parameters are calculated anyway). Gel parameters are found by difference, or from a transformation leading from the LPGF to the 'tie generating function'18. Those who use the complicated route of calculating such parameters by ad hoc probability arguments almost always make the mistake of neglecting correlations in the gel structure.

Thus $\alpha_{\text {sol }}$, but paradoxically not $\alpha_{\text {gel }}$, is the mean of a random link probability distribution. For example, the sol units of our random $f$-functional model do follow the random Bernoulli or binomial distribution, i.e. a unit chosen at random in the sol has the chance 


$$
p(i)=\left(\begin{array}{l}
f \\
i
\end{array}\right) \alpha^{i}(1-\alpha)^{f-i}
$$

of bearing $i$ reacted functionalities (links). There is clearly no analogy with the gel fraction in this respect, since the corresponding Bernoulli distribution with $\alpha \equiv \alpha_{\text {gel }}$ would merely split again into a sol fraction and a gel fraction; in particular, this distribution generates a fraction $p(0)=\left(1-\alpha_{\mathrm{gel}}\right)^{f}$ of totally unreacted monomer, which clearly has no place in the presumed gel fraction. Similarly for vulcanizates, the length distribution of chain segments between cross-links about $\bar{M}_{\mathrm{c}}$ is never a Flory (most probable) distribution in the gel either. This general paradox is resolved by reference to the statistical heterogeneity of the sample: for all calculations of gel parameters, which are attempted by excluding the sol from the calculation first and using $\alpha_{\text {gel }}$ directly. it would be essential to use a conditional probability distribution, i.e. conditional on applying to the gel. Then correlations of infinite range ${ }^{19 a}$ are bound to feature in such a conditional distribution. The conditional chance of finding a free functionality at one point of a supposed gel tree would be zero if all paths radiating from its bearer unit finally die out, and this can only be ascertained by following each branch to its bitter end, if any, at infinity. Fortunately, however, calculations involving $\alpha_{\text {gel }}$ as a parameter in this way are never necessary. In fact, no statistical technique is yet known ${ }^{19}$ for generating long-range statistics of the gel without contamination by sol : the correct and exact theory lumps sol and gel together but introduces the extinction probability $v$. Instead of requiring us to trace an infinity of paths for an infinite distance, the extinction probability obeys a recurrence relation, from which it is calculated (see equation 14). The second and third gel paradox concern genuinely three-dimensional effects: the 'Malthusian packing paradox' ${ }^{19}$ and the wrongly predicted 'cyclization explosion'20.

\subsection{Malthusian packing paradox}

Malthus greatly influenced Darwin with his observation that while family trees increase geometrically, the food supply for the members increases only in arithmetic progression. Similarly, the molecular family tree of a gel, by the geometrical progression of its members from generation to generation, outstrips - not its food supply — but the available space for accommodation. For our random $f$-functional model, when we trace the molecule from an arbitrary unit chosen as root of its tree (Figure 4), the number $N_{r}$ of members of generation $g_{r}$ increases as

$$
N_{r}=f \alpha\{(f-1) \alpha\}^{r-1}
$$

Thus the root on $g_{0}$ has $f$ functionalities, each with chance $\alpha$ of conversion to a link to an offspring on $g_{1}$ : the mean number of members on $g_{1}$ is $N_{1}=f \alpha$. Each of the members of $g_{1}$ in turn has on average $(f-1) \alpha$ offspring on $g_{2}$, so the total number of grandchildren on $g_{2}$ of the root-member is on average $N_{2}=f \alpha\{(f-1) \alpha\}$ and the total number of great grandchildren on $g_{2}$ is on average $N_{2}=f \alpha\{(f-1) \alpha\}^{2}$. If we proceed in this way, equation (7) is proved. Of course, the shells of members of successive generations in the roughly spherical-branched molecule find accommodation in three-dimensional 


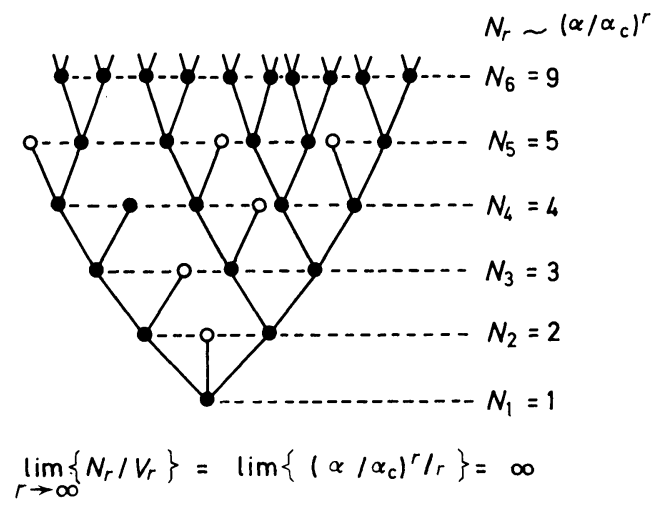

Figure 4. Illustration of the Malthusian Packing Paradox. A gel molecule is shown as a rooted family tree, with: $\bullet$ repeat units, $\bigcirc$ free functionalities. The number $N_{r}$ of repeat units increases (subject to statistical fluctuations) exponentially with the number $r$ of generations

space restricted to a volume $V_{r} \sim r^{2}$. Since after the gel point $(f-1) \alpha>1$, we have the limiting density of packing:

$$
\lim _{r \rightarrow \infty}\left(N_{r} / V_{r}\right)=\lim \left(c^{r} / r^{2}\right)=\infty \text {. }
$$

In chemical parlance, such structures are impossible because of steric hindrance. Real three-dimensional structures must obey the asymptotic law $N_{r+1} / N_{r} \rightarrow 1$ as $r \rightarrow \infty$. Diamond, for instance, does obey it. If we place one carbon as root on $g_{0}$, its four neighbours on $g_{1}$, its twelve second neighbours on $g_{2}$, and so on, the family 'tree' has $N_{r+1} / N_{r} \rightarrow 1$ (from above) because the presence of rings in the diamond graph leads to extensive sharing of offspring (as by intermarriage of second cousins in a genetic tree). Although $\alpha / \alpha_{c}$, or similar indices of branching in more complex network forming systems such as vulcanizates, can locally exceed unity, its average over an infinite graph cannot. Theories of networks are nevertheless based on tree-like models of average or crosslinking indices larger than unity, and the correct theories work well in practice. This paradox is resolved when the effects of cycles are taken into account correctly (see below).

First we point out how simply we can obtain Stockmayer's formula ${ }^{2}$ for the weight average degree of polymerization of a random $f$-functional polycondensate from equation (9). $D P_{w}$ is the mean number of units in a tree obtained from the choice of a unit at random which forms the root on generation $g_{0}$ (Figure 4). Now the mean of a sum is always the sum of the means. The mean number of units in a tree borne by a randomly chosen unit is merely $\sum_{0}^{\infty} N_{r}$ the sum of the mean number of units on $g_{0}(=1)$, on $g_{1}, \ldots$ etc. Thus from equation (7) we have

$$
D P_{w}=1+f \alpha \sum_{r=1}^{\infty}\{(f-1) \alpha\}^{r-1}
$$




$$
=(1+\alpha) /\{1-(f-1) \alpha\} \quad \text { (Stockmayer) }
$$

merely by summing the geometric series. The gel point condition of Flory follows :

$$
D P_{w} \rightarrow \infty \text { if } \alpha \uparrow 1 /(f-1) ; \quad \text { i.e. } \alpha=1 /(f-1)
$$

The question of ring-chain competition led Stockmayer ${ }^{2}$ to one slight criticism of Flory's gel theory: 'it may readily be demonstrated that the method of Flory predicts the occurrence of intramolecular reactions in the gel'- -since, indeed, in Flory's theory $\alpha_{\text {gel }}$ rises above $2 / f$ immediately after gelation. 'Quite aside from the fundamental logic involved in Flory's procedure', Stockmayer continued, '... it cannot be rigorous, for it predicts a very definite number of cyclic linkages in the system for any chosen total extent of reaction $\alpha$, though the initial assumptions do not specifically treat such structures in any way.' Flory's implicit assumption, however, was the intuitively sound one made more explicit in the citation from James and Guth (see later): the rings in the gel arise from collisions under the mass law of pairs of functionalities which move like free particles.

Stockmayer took the total exclusion of rings, even from the gel, seriously and arrived at the conclusion that $D P_{n \text {, sol }}$ was constant irrespective of conversion $\alpha>\alpha_{\text {get }}$. Whittle ${ }^{21}$ recently calculated the same rigorous model in more detail, and showed that the whole sol distribution, not only its mean $D P_{n \text {, sol }}$, is invariant. But this is not physically realistic in flexible gel molecules, and Flory's model•or, even better, its generalization to the spanning-tree approximation for ring-chain competition described below is excellently supported by experiments.

The spanning-tree model assigns to the intermolecular process one fundamental rate constant $k_{x}$, and to the intramolecular processes in the sol one fundamental rate constant, $\mathrm{k}_{\sigma}$. The actual rate of intermolecular reactions is proportional to $k_{\alpha}(1-\alpha)^{2}$. The actual rate of forming a ring-closure which unites two sol functionalities separated by a (minimum) path of $x$ atoms is proportional to $k_{\sigma} F_{x} x^{-\frac{3}{2}}$. Here $F_{x}$ is the mean number of free functionalities at distance $x$ within the graph; the factor $x^{-\frac{3}{2}}$ comes from Gaussian chain statistics in three dimensions. The links already formed may be assigned a constant rate of scission proportional to their concentration; however, we shall equate it to zero in order to treat at present only the irreversible kinetic process. Gordon and Scantlebury ${ }^{22}$ generalized this model to allow for cocondensation (e.g. of adipic acid and pentaerythritol), and for the first-shell substitution effect of reacted links on the reactivity of neighbour functionalities. They wrote down the necessary differential rate equations and introduced into the statistical treatment one asymptotically exact approximation, later called the spanning-tree approximation ${ }^{23}$. Asymptotic correctness means that the relevant first-order quantities, especially the displacement $\delta \alpha_{c}$ of the critical intermolecular conversion from the classical model, become exact as the fraction $\sigma$ of functionalities reacted intramolecularly (to form rings) goes to zero. The equations were solved by computer and fitted successfully to experiments, later extended to other substrates, especially longer-chain dicarboxylic acids ${ }^{24}$. The effect of dilution with inert solvents gives rise to increase in $\sigma$ at fixed $\alpha$-in particular, at $\alpha_{\mathrm{c}}$. The Stockmayer plot 
of $\left(\alpha_{c}+\sigma_{c}\right)$ against reciprocal concentration has been used for independent confirmation of the model parameters ${ }^{22,25}$.

\subsection{The cyclization explosion paradox}

It is a paradoxical feature of the model just described that the over-all cyclization rate would seem to diverge to infinity at the gel point. The argument resembles that for the Malthusian paradox. The cyclization rate is the sum of rates of formation of rings of all sizes. The rate of forming a ring of size $x$ repeat units is reduced by the factor $x^{-\frac{3}{2}}$ through Gaussian statistics, which opposes the chance of two functionalities separated by a chain of length $x$ units meeting by random diffusion. However, the rate of formation of rings of size $x$ is also proportional to the number, $F_{x}$, of free functionalities available at distance $x$ to a given unit in the branching structure, equal to

$$
F_{x}=(f-1)(1-\alpha) N_{x}
$$

It follows from equation (7) that this quantity again diverges exponentially as soon as the gel point is passed, even when multiplied by the Gaussian weighting of $x^{-\frac{3}{2}}$. As defined, the number of reaction partners for ring formation diverges so catastrophically immediately after the gel point that the situation is not remotely saved by the Gaussian damping factor, just as the packing problem is hardly touched by the factor $x^{-2}$ arising from the increasing space accommodation.

The error in the formulation, which leads to this catastrophe, can be corrected rigorously and exactly ${ }^{20}$. The rate equations have to be corrected for an implicit over-counting of the intramolecular reaction steps within the gel (gelgel steps), relative to sol-gel and sol-sol reaction steps.

Not only are such gel-gel steps included in the $k_{\sigma}$-term (and to such an extent that they cause this term to give a divergent rate), but they are also included in the $k_{\alpha}$-term. In the region just after the gel point, it makes the best physical sense to accept the contribution of gel-gel steps to the $k_{\alpha}$-term completely, and to cancel out completely their contribution to the $k_{\sigma}$-term, thus removing the divergence. This amounts to treating gel-gel steps as bimolecular collisions governed merely by the law of mass action, and not in terms of Gaussian statistics of the sub-chain linking the two functionalities concerned.

The automatic inclusion of the gel-gel steps in the $k_{\alpha}$-term arises as follows. In accord with the mass law, this term treats the chance of a given functionality undergoing reaction as proportional to the total concentration of partner functionalities available for reaction in the system as a whole. If, and only if, the given functionality resides on the gel, a finite fraction of the number of partner functionalities in the system as a whole resides on the same molecule as the given functionality (in this way intramolecular steps creep into the rate term designed for intermolecular steps) ${ }^{20}$.

The physical plausibility of letting gel-gel linking steps be represented by the mass-action contribution already automatically included in the usual rate equation, and thus disallowing any part to be played by configurational chain statistics, has been expounded before ${ }^{20}$. A long quotation from the fundamental paper by James and Guth ${ }^{26}$ was given which culminates in the clear statement: 'the relation of these chain elements is then almost the same as if 
they were separate molecules in an ordinary liquid'. The plausibility, in particular, of avoiding, by this formulation of the theory, the unreasonable divergence of the cyclization rate at $\alpha=\alpha_{\mathrm{c}^{+}}$may be demonstrated as follows. The divergence arises because the number of reaction partners increases exponentially with the length of the paths in the branching molecular tree which we trace (Figure 5) from a given free functionality to such partners. It

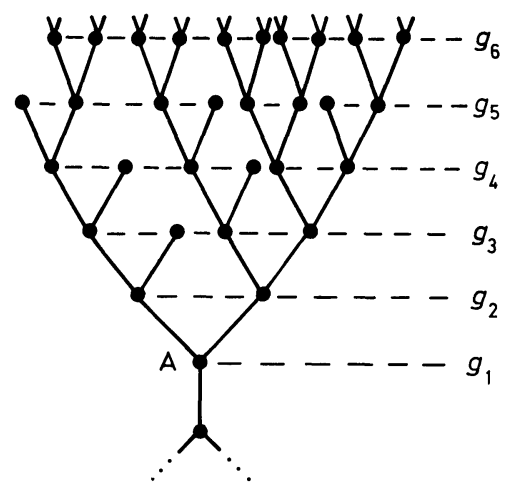

Figure 5. Diagram to explain the definition of extinction probability $v$. The base of the tree a link chosen at random. rather than a point (repeat unit) as in Figure 4

would be wrong to imagine that all these potential reaction partners are in true competition for cyclization with our free functionality merely by virtue of micro-Brownian configurational changes subject to Gaussian statistics. In reality. a more or less random selection of these potential partners will happen to lie close enough to collide with the free functionality in a reasonable time while the vast majority lie far away in three-dimensional space, and are often separated by many active network junction points along the path in the graph from the free functionality. In practice, they will never achieve a collision with it. So the potential reaction partners are rather sharply divisible into two classes: members of the first class happen to lie close to the free functionality and are practically unaffected by configuration constraints (because the intervening paths along the graph are so long); the other class never has a chance. The reactive class reacts by collisions under a mass-law régime. This régime implies that there are many unsuccessful collisions before a successful one which leads to a cycle being formed. As the reaction proceeds well beyond the gel point. however. the exhaustion of free functionalities and restrictions in motional freedom cause a transition to a régime of diffusion control, in which every collision is successful. The inapplicability of Gaussian chain statistics (based on unperturbed chain dimensions) to collisions between two functionalities belonging to the active network deserves emphasis. For example, by an attempted application of such unperturbed Gaussian statistics to the kinetics of the formation of inactive chain loops in the network. the extent of this kind of wastage of cross-links in competition with effective cross-linking was rather overestimated in a recent paper (see reference 27). 
To implement the cancellation of the unwanted Gaussian chain cyclization effects from the $k_{\sigma}$-term in the rate equation requires a relatively trivial correction: essentially $F_{x}$. the mean number of reaction partners for closing an $x$-ring with a free functionality. must be generalized from

$$
f \alpha\{(f-1) \alpha\}^{x-2}(f-1)(1-\alpha) \text { to } f \alpha v\{(f-1) \alpha v\}^{x-2}(f-1)(1-\alpha)
$$

(cf. equations 7 and 12). Here the extinction probability $v$ is introduced. which is unity up to the gel point but $<1$ thereafter. We turn to explaining this parameter in the next section. Experimental verifications of the kinetic scheme based on extinction probabilities. analogous to equation (13). have been given ${ }^{20,25}$.

\section{THE EXTINCTION PROBABILITY $v$}

Consider a trifunctional random (and therefore ring-free) polycondensate at conversion $\alpha$. e.g. polyetherified 1.3.5-trimethylol benzene ${ }^{9}$. Choose one of its ether links at random. and choose at random one of the two ways of orienting it vertically on the page. The repeat unit A (Figure 5) which we are bound to find at its upper end bears two further functionalities which we direct upwards, and we consider the molecular sub-tree that has its root (lower end) at $\mathrm{A}$ : if this sub-tree is finite. we say that our chosen link becomes extinct in the chosen (upward) direction; if it is infinite, our chosen link forms a tie in this direction (see later). The probability $v$ of extinction. or $1-v$ of nonextinction, is found from the assumptions of the random condensation model, by writing down and solving a recurrence relation.

The sub-tree rooted at $\mathrm{A}$ is extinct, with probability $v$, if and only if the two functionalities pointing upwards from $A$ bear at most finite sub-trees. The chance $(1-\alpha+\alpha v)$ that one of them bears at most a finite sub-tree is made up of two parts: a part $(1-\alpha)$ that it is found unreacted. which means immediate extinction. plus a chance $\alpha v$ that it has reacted (chance $\alpha$ ) to form a link which in turn leads only to a finite sub-tree (with chance $v$ ). The chance that both upward functionalities lead to extinction is thus $(1-\alpha+\alpha v)^{2}$. so that

$$
v=(1-\alpha+\alpha v)^{2}
$$

To solve this recurrence relation for $v$ does not even demand the solution of a quadratic equation. All equations in $v$ have as one root $v=1$. as is obvious by inspection in the case of equation (14). Removing this root and solving the resulting linear equation gives

$$
v=[(1-\alpha) / \alpha]^{2}
$$

This quantity $y$, so simple to calculate in a simple case, provides the unique and universal key for calculating the sol and gel fractions and their statistical parameters. Its role in equation (13) is now clear: wherever the chance $\alpha$ of finding a link (as distinct from the chance $1-\alpha$ of a free functionality) occurs. we multiply it by $y$, to implement our model postulate that our calculation is restricted to the population of molecules of finite size. It is understood that we always choose $v$ as the smallest positive root of a defining equation 
such as (13) or (17). Up to the gel point, this is always $v=1$, so equations (13) and (17) are true before, at and after the gel point, a fact appreciated by electronic computers.

\section{SCANLAN-CASE EANCs}

Scanlan ${ }^{6}$ and Case $^{7}$ showed how to define an elastically active network chain (EANC) of a tree-like gel. First, an active junction point is a point from which at least three 'ties' (links leading to infinite sub-trees) radiate, since in three-dimensional space a network point would relax after an affine transformation of the specimen, unless it was tied to the surface by three independent routes. (In the statistical model the surface is, of course, projected to infinity.) An EANC is a chain segment which has active junction points only at its two ends. Figure 6 shows how this definition of an EANC

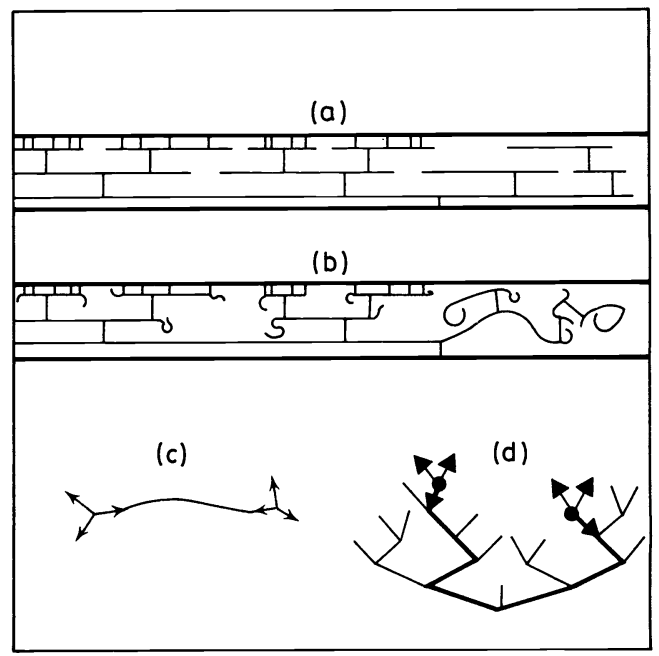

Figure 6. (a) Schematic sketch of section of vulcanizate gel of chains and crosslinks, immediately after stretching. (b) The same, after relaxation. Note that not only chain ends have relaxed, but also more complex loosely attached material, such as those shown on the right. (c) Definition of elastically active network chain. Each of the two network junction points must have at least three 'ties' (shown by arrows). A tie is a link from which a path can be drawn to the surface of the specimen along the gel molecule. (d) An active network chain (heavy line) in a trifunctional polycondensate (cf. Figure 1). Note that all the branches (thin lines) attached along the active chain can relax after stretching

applies naturally to rubbery networks made either by polycondensation of small units or by vulcanization of primary chains.

The reason why the tree-like gel model gives such an excellent account of the properties of a real network containing more and more cycles as $\alpha$ increases beyond $\alpha_{c}$ is simple to state. If a link attached to a network junction point is a tie, so that at least one path to infinity passes from the point through the link, then cascade statistics assures us that there will be an exceedingly large number of paths which can be traced starting from that point, through that 
link and on through the branching structure. In the real structure some of these paths will be abortive, because some of the links which we allowed to form as bimolecular steps (both in the kinetics and in the statistical model) really have the character of formation of large cycles in the gel. But it is most unlikely that all the almost innumerable paths would terminate prematurely in cycles, thus requiring the formal reclassification of a tie as a non-tie. The calculated concentration of ties and of EANCs will be exact within experimental error.

To give a feeling for the simplicity and power of this Scanlan-Case EANC. we shall once again do the calculation for the simplest case, the trifunctional random polycondensate. The number $N_{\mathrm{e}}$ of EANCs per repeat unit, to which the elastic modulus is proportional, is found as the number of active junction points per repeat unit, times $\frac{3}{2}$. This factor $\frac{3}{2}$ occurs because (a) each active junction point is here attached to three ties exactly, which lead to one EANC each; and (b) each EANC is attached to two active junction points at its ends, and so we divide by two to correct the overcount.

The number of active junction points per repeat unit is merely the fraction of repeat units which are active junction points, viz. $\{\alpha(1-v)\}^{3}$. (Each of the three functionalities has a chance $\alpha$ of being reacted, and-if reacted-a chance $(1-v)$ of having formed a tie.) Thus we have, for trifunctional random condensates,

$$
N_{\mathrm{e}}=\frac{3}{2}\{\alpha(1-v)\}^{3}=\frac{3}{2}\{2 \alpha-1 / \alpha\}^{3}
$$

by using equation (15). The first equality holds for all $\alpha$. with $v=1$ (i.e. $N_{\mathrm{e}}=0$ ) up to the gel point, $\alpha_{\mathrm{c}}=\frac{1}{2}$; the second equality makes physical sense only after the gel point. Strella and Bibeau ${ }^{28}$ obtained the same formula (in an algebraically complicated but equivalent ${ }^{29}$ form) and confirmed its essential features by experiments on a model phenolic resin (Figure 7).

The generalization from tri- to $f$-functional random polycondensates of

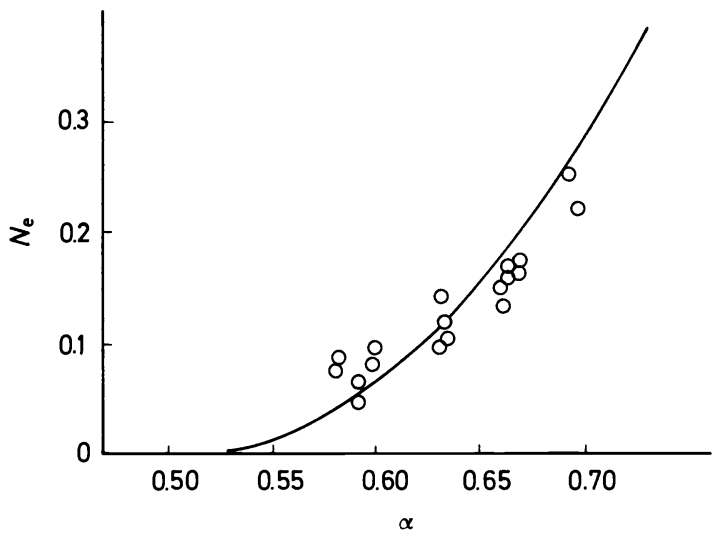

Figure 7. Fit by Strella and Bibeau ${ }^{28}$ of their measurements by equilibrium swelling of $N_{\mathrm{e}}$ against conversion $\alpha$ for a model trifunctional phenol/formaldehyde resin. The curve is calculated from equation (16) 
equation (14) is obvious; $v$ is the lowest positive root of

$$
v=(1-\alpha+\alpha v)^{f-1}
$$

In generalizing equation (16) for the relative number of EANCs. we must remember that active junction points may now have $3.4 \ldots . . f$ ties. A little calculation ${ }^{18}$ gives

$$
N_{\mathrm{e}}=3 \alpha(1-v)^{2}(1-\beta) / 2
$$

where

$$
\beta \equiv(f-1) \alpha v /(1-\alpha+\alpha v)
$$

Turning to the case of randomly cross-linked linear primary chains of any normalized number-fraction distribution function $n_{x}$, with number-average $D P_{n p}$, we have the following generalization of equation (17):

$$
v=\sum_{x} n_{x} x(1-\alpha+\alpha v)^{x-1} / D P_{n p}
$$

It is well known that the gel point is given by

$$
\alpha_{c}=1 /\left(D P_{w p}-1\right)
$$

where $D P_{w p}$ is the weight-average $D P$ of the primary chains. If. following the usual cascade formalism, we introduce the definition

$$
F_{0}(v)=\sum_{x} n_{x}(1-\alpha+\alpha v)^{x}
$$

then the completely general formula of Dobson and Gordon ${ }^{18}$ for the number of EANCs per primary chain may be quoted, thus:

$N_{\mathrm{e}}=\left[\gamma\left(1-v^{2}\right)-2\left\{1-F_{0}(v)\right\}\right]\left\{\gamma(1-v)(1-2 v)+1-F_{0}(v)\right\} / \gamma(1-v)^{2}$

with

$$
\gamma \equiv \alpha \bar{D}_{n p}
$$

This formula generalizes and frees from approximations numerous results obtained by lengthy calculations of individual cases in a number of papers $^{6,7,30-32}$. Three regions of this equation, and of equation (18), are important for studies of elasticity, swelling, etc. : (1) the region immediately following the gel point. (2) the region of intermediate degrees of crosslinking which follows it, and then (3) the asymptotic domain of high crosslinking. These regions are discussed below.

\subsection{The gel point as a fifth-order Ehrenfest transition*}

For random (ring-free) systems, equations (14)-(23) apply equally to the equilibrium case (crosslinking rate $=$ splitting rate) and the irreversible case (splitting rate $=0$ ). We shall now turn to the thermodynamic nature of the reversible transition, for which the equilibrium conversion is controlled by

* The transition was wrongly stated to be in most cases of order 4 in a contribution by the author to the Discussion of the Faraday Division on Gels and Gelling Processes. 
temperature or pressure, etc. Stockmayer called it a phase transition, and compared it to the condensation of a vapour; cf. Gibbs et al. ${ }^{33}$. We shall show that it is always an Ehrenfest transition of the fifth order. It is easy to show qualitatively how this comes about. According to classical rubber elasticity theory, Young's modulus $Y$ is a quantity proportional to a second derivative of the free enthalpy. The modulus is not discontinuous as the equilibrium conversion $\alpha$ is driven through its critical value $\alpha_{c}$, and exactly three differentiations of the modulus are necessary to reach a quantity which is discontinuous at the gel point, making a total of five differentiations starting from the free enthalpy. The three differentiations of the modulus arise because the modulus is proportional to $N_{\mathrm{e}}$, the relative number (concentration) of EANCs. According to the Scanlan-Case definition, an EANC links two active branch points. i.e. two branch points with at least three ties (links through which paths continue to infinity). But initially, as active branch points come into existence immediately after the gel point, they will almost always have exactly three ties. since the chance of having more ties will be an infinitesimal of higher order (or zero for $f=3$ ). Moreover, the chance $\alpha(1-v$ ) that a functionality leads on to a tie is continuous through the gel point, while its derivative is discontinuous (Appendix B), i.e. $\partial v / \partial \alpha$ (like $\partial S / \partial \alpha$, where $S$ is the sol fraction) is zero for all $\alpha<\alpha_{\mathrm{c}}$, but jumps to a finite value at $\alpha_{\mathrm{c}}$. Accordingly, $N_{\mathrm{e}}$ is found to be proportional to $(1-v)^{3}$ (e.g. equation 16) at $\alpha=\alpha_{c^{+}}$, because a branch point will typically acquire three ties to become active, so that $\mathrm{d}^{3} N_{\mathrm{e}} / \mathrm{d} \alpha^{3}$ and $\mathrm{d}^{3} Y$ / $\mathrm{d} \alpha^{3}$ are discontinuous. Some relevant results are given for various chemical systems (molecular graphs) in Appendix B; full calculations will be submitted elsewhere.

This has substantial practical and theoretical importance. The curve of $Y$ against $\alpha / \alpha_{c}$ always starts at $\alpha=\alpha_{c}$, like a cubic curve tangent to the $\alpha / \alpha_{c}$ axis. It is true that measurements close to the gel point are not accurate enough to verify the cubic law with great precision, but the general shape has been well verified $^{35}$ under kinetic conditions in a great diversity of systems (Figures 7-10). It is sometimes argued that a fifth-order Ehrenfest transition is almost

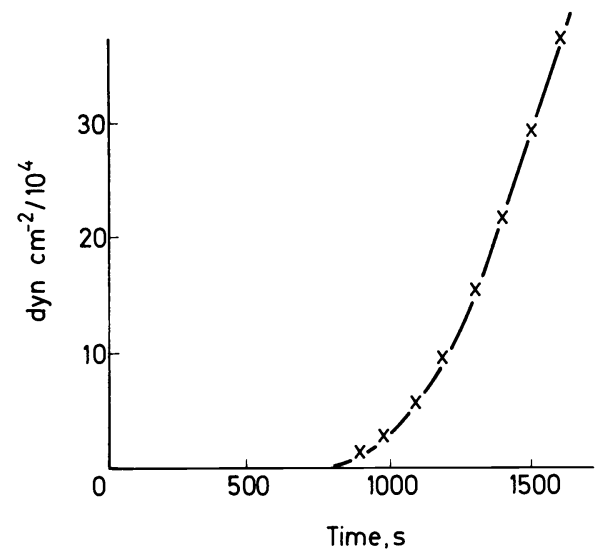

Figure 8. Measurements ${ }^{34}$ of shear modulus versus curing time for a 'Beetle' unsaturated polyester resin 


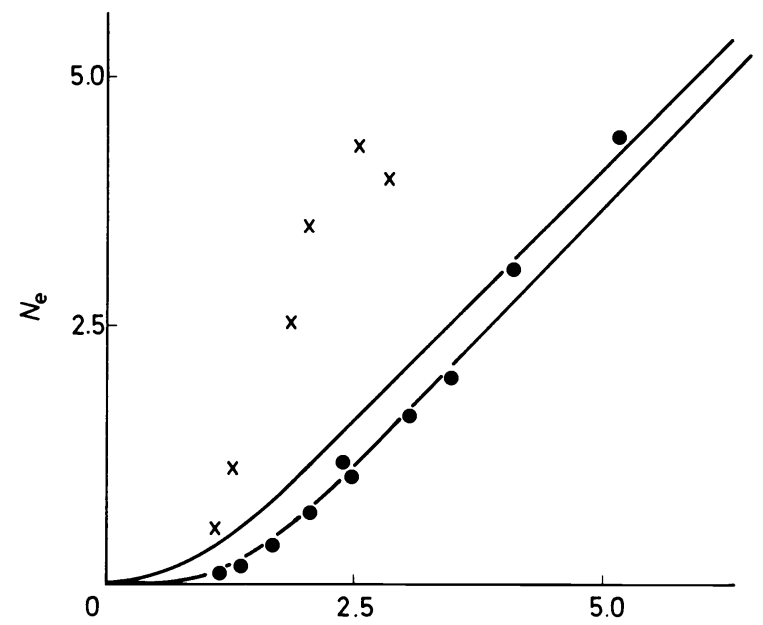

Figure 9. Comparison (see reference 37) of experimental results by Meissner and Kuchařík of concentration $N_{\mathrm{e}}$ of active network chains per primary chain against crosslinking index $\gamma$ for two polybutadienes. The lower curve is calculated to fit the primary distribution $(\varepsilon=0.375)$ of the solid circles and does fit well; the upper curve that $(\varepsilon=0.688)$ of the crosses (poor fit)

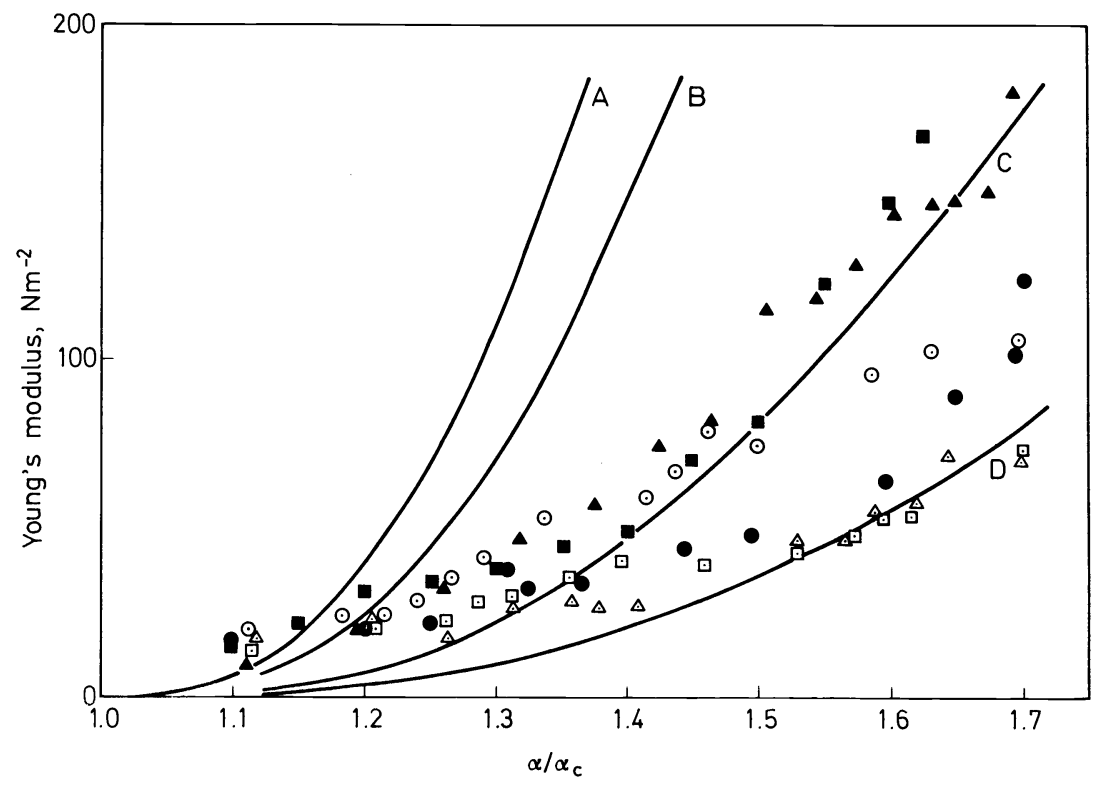

Figure 10. Plots of measured Young's modulus against relative conversion (from optical rotation measurements ${ }^{10}$ ), for six runs at two different temperatures. Each run was carried out on a freshly made solution. Gel times at $26.6^{\circ} \mathrm{C}: \triangle .61 \mathrm{~min} ; \square, 60 \mathrm{~min} ; 0,60 \mathrm{~min}$. At $26.9^{\circ} \mathrm{C}: \Delta .76$ $\min ; \boldsymbol{C}, 80 \mathrm{~min} ; \bullet, 77 \mathrm{~min}$. Four theoretical plots (equation 31); A, $x=6$ sites per primary chain, and $n=2$ primary chains involved in each helix; $\mathrm{B}, x=20, n=2 ; \mathrm{C}, x=8, n=3 ; \mathrm{D}, x=8$. $n=4$ 
meaningless to an experimentalist and in practice unverifiable, but this is a serious misunderstanding. It is not claimed that one can ever determine the precise order of a transition by making measurements exactly at that point. Other phenomena will obscure the results there, e.g. premelting in a first-order case. But successful measurements at a small distance from the point can establish the formal contribution to the order of the transition by a dominant mechanism which is of interest, as (for instance) in the case of the DebyeScholte extrapolation ${ }^{36}$ to a spinodal point.

Experimental verification well within the cubic range is afforded by the system decamethylene glycol/benzene 1,3,5-triacetic acid $^{20}$, although the proportionality constant is about three times too high, which remains unexplained ${ }^{10}$. Verification of equation (18) is excellent for the phenolic resin of Strella and. Bibeau ${ }^{28}$, as shown in Figure 7. The experimental range is rather beyond the scope of the purely cubic approximation. Figure 8 presents data $^{34}$ on a cross-polymerized polyester resin which shows the initial upturn qualitatively. Figure 9 shows one convincing fit of theory to data by Meissner and Kuchařík on natural rubber vulcanizates ${ }^{33}$, with the same initial upturn which should start as a cubic equation. In the next section, Figure 10 illustrates the same theory for an aqueous gelatin system.

The initial cubic law has thus been at least approximately verified directly. or by extrapolation to the measured gel point, essentially without adjusting parameters (except for some doubt concerning the proportionality constant) for very diverse systems. This tends to confirm that three units are contributed to the order of reversible gel transition by the long-range correlations through the factor $(1-v)^{3}$. Theoretically the high order of the Ehrenfest transition is the result of an enormous co-operativity effect, depending on three independent and branching paths which run through the whole substance of the example.

\subsection{Intermediate degrees of crosslinking}

For network formation by random linking processes of long primary chains, the plot of $N_{\mathrm{e}}$ or Young's modulus $Y$ against the crosslinking index 8 (see equation 24) must always have an inflection point, i.e. the plot is sigmoid. This follows geometrically from the emergence of the plot with zero slope at the gel point, together with the asymptotic approach (see below) of the plot at high $\alpha / \alpha_{c}$ to a line from its underside. It has been shown ${ }^{37}$ that for vulcanization of any primary distribution the slope at the inflection point $\leqslant 1.5$. and in practice it is usually around 1.2 .

\subsection{At high crosslinking}

At high crosslinking $(\alpha \rightarrow 1)$. the plot of $N_{\mathrm{e}}$ against $\gamma$ for long primary chains of any distribution approaches asymptotically from below the line:

$$
N_{\mathrm{e}} \sim \gamma-1
$$

In this domain of high crosslinking, $N_{\mathrm{e}}$ becomes all but proportional to the number, $\gamma$. of crosslinked units per number-average primary chain, although the term -1 represents an ineluctable 'wastage' of crosslinks arising from one free end of the primary chains. Of course, the plot terminates when every unit is crosslinked $\left(\gamma=\overline{D P}_{n p}\right)$. For the formation of networks by end-linking of homodisperse three-functional star units. the simple equation (16) is applic- 
able. In this case, the plot of $N_{\mathrm{e}}$ against $\alpha / \alpha_{\mathrm{c}}$ remains concave upwards ${ }^{18}$ to its terminal point $\left(\alpha / \alpha_{c}=2\right)$, at which the number of EANCs per star unit is $\frac{3}{2}$. and the terminal slope is

$$
\left\{\partial N_{\mathrm{e}} / \partial\left(\alpha / \alpha_{\mathrm{c}}\right)\right\}=\frac{9}{4} . \quad\left(\alpha / \alpha_{\mathrm{c}}=2\right)
$$

Because of the high sensitivity of $N_{\mathrm{e}}$ to $\alpha / \alpha_{\mathrm{c}}$, the elastic modulus could be useful in studying the diffusion-controlled death-throes of the cross-linking reaction (as $\alpha \rightarrow \alpha_{\text {final }}<1$ ), but star units with long flexible branches would have to be employed.

\section{Discussion}

Much current theorizing concerning rubbers and jellies (i.e. weakly rubbery materials) attempts to graft refinements on too roughly formulated basic models. The gel transition, one of the most highly co-operative phenomena known, exerts its statistical effects not only in the critically branched state of viscoelastic behaviour in the close vicinity of the gel point, but even at what are considered asymptotically high degrees of crosslinking. Although an average quantity such as $\bar{M}_{\mathrm{c}}$ (the number-average chain length between crosslinks in vulcanizates) appeals to intuition, it is an undesirable ingredient of any theory, and especially so if refinements of basic theory are aimed at to cover effects such as chain loops or entanglements. Authors were forced to introduce chain-end corrections in addition to $\bar{M}_{\mathrm{c}}$ from the early days of network theory; but calculations based on the correct Scanlan-Case-type EANCs show how poorly such corrections deal with inactive material belonging to the gel. In addition, the actual 'correction' factor applied ${ }^{27}$ is usually too large by a factor of two. A network theory not based on the extinction probability $v$ (or Charlesby's approximation thereof by the sol fraction $S$ for long-chain vulcanizates) is not worth considering today. Apart from the simplification introduced by the extinction probability into the mathematical formulae, this concept immediately applies to all kinds of networks however synthesized. If the reader cares to look at the simple formula (16), essentially verified by swelling equilibria (Figure 7), he might like to consider what meaning he could possibly attach to $\bar{M}_{\mathrm{c}}$ in such a network. Other parameters beside $\bar{M}_{\mathrm{c}}$ should be discarded or recalculated on the proper statistical basis. Dušek ${ }^{38}$ showed that, even after moderately heavy crosslinking, the statistical value of the effective functionality $f_{\mathrm{e}}$ of junction points (i.e. the mean number of ties on an active junction point) falls appreciably below the value 4; e.g. in the vulcanization of long chains $f_{\mathrm{e}}$ is only 3.5 when on average each chain carries 10 crosslinks. Chemically, of course, each crosslink has functionality 4 , but in network theories only active branches must be counted. In another paper, the same author ${ }^{39}$ developed the theory to expose the appreciable sensitivity of network properties to first-shell substitution effects (FSSE, of a functionality on the reactivity of a neighbouring one) in polycondensates. The occurrence of FSSE effects in the esterification of pentaerythritol with polybasic acids ${ }^{22}$ has recently been verified by kinetic and GPC analysis on monofunctional carboxylic acid analogues ${ }^{40}$. As regards systems derived by randomly crosslinking primary chains, a routine method has been published ${ }^{41}$, for calculating the $z$-average particle scattering factor $P_{z}$ (up to gelation. or in the sol thereafter), starting from any given primary 
chain distribution. From $P_{z}$ the Zimm plot leads to $M_{w}$ and $\left(\overline{R^{2}}\right)_{z}$. The theory underlying these calculations is based on the assumption of Gaussian or random-flight statistics for all sub-chains (paths) in the system. and satisfactory experimental verification has been forthcoming.

\section{A MODEL FOR THE NETWORK IN WEAK AQUEOUS GELATIN JELLIES}

We briefly report some more recent results of Judd. to supplement a report of preliminary measurements on the gelation of aqueous gelatin ${ }^{10}$ and the development of an equilibrium Young's modulus $Y$ (Figure 10) by the resulting jelly. Only brief experimental details need be given (see below), because the treatment of the data sets this work apart from the situation typical for gelatin research. Normally, the study of the physical properties of gelatin systems needs careful control over such variables as source, preparation. thermal treatment, $\mathrm{pH}$, electrolyte content, etc. Our sample solutions were. of course, prepared from a well-characterized gelatin and carefully handled, but the reduced plots (viscosity $\eta$ or $Y$ against $\alpha / \alpha_{c}$ ) are claimed to render results in any case very largely independent of the usual troubles. This is the power of reduced plots based on critical points generally: e.g. the WLF transform. based on $T_{\mathrm{g}}$, superposes properties of the most chemically diverse glass-forming substances. Similarly, theory suggests that a gelatine solution which gels at all (under isothermal conditions) will give a reduced plot characteristic in general shape for all gelling systems (cf. Figures 7-9); if it does not differ too radically from the range of condition here employed, it is expected to fit approximately to the theoretical plot $\mathrm{C}$ in Figure 10, which is quite insensitive to adjustments in relevant parameters.

\section{Experimental details}

The viscosity $\eta$ of a quenched 5.7 per cent aqueous solution of Ilford SC200 gelatin (taken to consist essentially of homodisperse $\alpha$-chains of molecular weight 105000 ) was followed isothermally to the gel point in a moving-sphere microrheometer, which also allowed $Y$ to be charted after gelation. $Y$ is plotted for six separate solutions at two temperatures against the relative conversion $\alpha / \alpha_{c}$ in Figure 10 . The latter quantity is deduced by assuming $\alpha$ to be proportional to the change in optical rotation, measured separately for two such solutions at the same concentration at $26.9^{\circ} \mathrm{C}$ against time. The specific volume was found to change proportionally to optical rotation. This calibration plot, and some of the data in Figure 10 have been previously ${ }^{10}$ published, together with some further experimental details.

\section{Model for gelation of gelatin}

The model fitted to the plot of $Y$ against $\alpha / \alpha_{c}$ in Figure 10 is as follows. A set of $n$ chains locally come together to form an $n$-fold helical junction zone. acting as crosslink among the set of primary chains concerned. For $n=2$, this is the familiar model for carrageenan, illustrated in Figure 1 of the recent paper by Bryce et al. ${ }^{42}$. The isothermal coil-helix transition of a given junction zone is assumed to be fast compared with the rate of nucleation of junction zones and to give rise to a constant contribution to the optical rotation or specific volume of the solution. Each primary chain has a constant 
number, $x$, of sites suitable for participating in a helical junction zone and the régime of crosslinking is random, i.e. there is no correlation in the chances of forming junction zones between any of the sites. A random network between the junction zones will thus be formed, and the usual tree-like graph model is readily applied. In particular, the gel point is found to be

$$
\alpha_{c}=\{(x-1)(n-1)\}^{-1}
$$

where the fractional conversion $\alpha$ of sites to junction zones is monitored by optical rotation.

Let $q$ denote the chance that a site on a given primary chain A does not participate in a junction zone in such a way that a path can be traced to infinity from that site, through that junction zone (i.e. not passing through other sites belonging to A). Then

$$
q=1-\alpha+\alpha v
$$

and the extinction probability $v$ obeys its standard recurrence relation. generalizing equation (14):

$$
v=q^{(x-1)(n-1)}
$$

Hence, $q$ can be calculated as a function of $\alpha$ for given $x$ and $n$ on a pocket calculator. The calculation can be continued to give (in a form simpler than the equivalent formula ${ }^{10}$ given before) the quantity proportional to Young's modulus

$$
N_{\mathrm{e}}=Y V_{\mathrm{mol}} / 3 g R T
$$

viz.

$$
N_{\mathrm{e}}=(1-q)^{x}-1+q^{x}-\frac{2(n-1)(1-q) q^{(x-1)(n-2)}}{x(1-q)^{(x-1)(n-1)}}\left[\frac{1-q^{x}}{1-q}-x q^{x-1}\right]^{2}
$$

Here $g$ is the front factor taken as $\frac{1}{2}$ on theoretical grounds and $V_{\text {mol }}$ the volume per mole of primary chains. calculated to be $1.8 \times 10^{6} \mathrm{ml}$.

The four plots in Figure 10, based on equation (31), show two surprising facts. When the reduced variable $\alpha / \alpha_{\mathrm{c}}$ forms the absicissa, the plots are (a) not very sensitive to the number of sites, $x$, per chain when $x>6$, but (b) remarkably sensitive to the number $n$ of chains per junction zone.

The scatter of the results in Figure 10 reflects the difficulties in measuring very low moduli. Superposition of the plots for two different temperatures is. however, satisfactory.

The moduli measured immediately after gelation are seen in Figure 10 to lie systematically above curve $B$, calculated from the relative critical conversions $\alpha / \alpha_{c}$ (found by optical rotation) via equations (27)-(31). This systematic deviation is not attributable to a horizontal misplacement of the data points arising from an error in the gel point $\left(\alpha / \alpha_{c}=1\right)$, since this point is indicated within 0.05 by the divergence of the viscosity and the emergence of a reasonable modulus. The cause of the inflated modulus values is assigned to the viscous component, which is noticeable in extremely weak gels, especially when the 'crosslinks' are easily reversible. (The displacement of the micro- 
sphere under the magnetic force was not always found to be fully recoverable just after gelation.) At conversions $\alpha / \alpha_{c}>1.4$ the fit to the theoretical plot $\mathrm{C}$ is thought to be reasonable, and the marked upswing of the data points observed in the polycondensate DMG/BTA seems to be absent in these gels. The upswing is traditionally assigned to 'physical crosslinks' due to entanglements. However, while such a contribution is theoretically expected in the dynamic behaviour at appreciable frequencies, the relevance of entanglements to true equilibrium moduli seems much less well founded in theory.

The parameter $x$, the number of sites per chain, was taken as eight in calculating curve $\mathrm{C}$ of Figure 10 from equations (30) and (31). Ferry ${ }^{43}$ proposed that $x=5$, and this value is also confirmed approximately-on obvious assumptions by comparing our experimental change in optical rotation $\left(\sim 33.5^{\circ}\right)$ with the known value for total renaturation of collagen $\left(\sim 275^{\circ}\right)$. Using equation (27), we thus find

$$
1 /(n-1)(x-1)=1 / 2(x-1)=33.5 / 275
$$

which yields $x \sim 5$. The difference between $x=8$ and $x=5$ causes only a small displacement downwards in the plot C (Figure 10), about 25 per cent initially (see equation B5). Such a displacement is hardly significant for our purpose - namely to confirm the applicability of the basic statistical theory of gelation and rubber elasticity to aqueous gels of gelatin. Despite the experimental scatter, since all the significant parameter values used in calculating plot $\mathrm{C}$ are independently supported by theory and other experiments, this purpose is broadly achieved. Further work is, of course, desirable, especially since the exploitation of the critical nature of the gel transition holds the promise of increasing the sensitivity of experimental tests of theory to most parameters which are of interest, while allowing unwanted interferences to be eliminated by the rescaling according to $\alpha / \alpha_{\mathrm{c}}$.

\section{ACKNOWLEDGEMENTS}

Sincere thanks are due to $\mathrm{Mr}$ M. Judd for the experiments cited and to Mr S. B. Dev for advice and computer calculations.

\section{AppendixA: Estimation of $\bar{x}_{\max , i}$ (Figure 2)}

Throughout Appendix A we consider an ensemble of an infinite number of reaction systems, each containing a fixed finite number, $N$, of repeat units, and a fixed conversion, $\alpha$, just below $\alpha_{c}$. The degree of polymerization, $x_{\max , 1}$ of the largest polymer molecule will vary appreciably from system to system. We derive an approximation to the mean, $\bar{x}_{\max , 1}$. over these statistical fluctuations, together with rather sharper error limits than suggested before. We note that

$$
N \int_{y}^{\infty}\left(w_{x} / x\right) \mathrm{d} x=\bar{n}
$$

Here $\bar{n}$ is the mean number of molecules per system of size larger than $y \equiv y(\bar{n})$. Using the asymptotic form of $w_{x}$ of equation (5) and the approximation 


$$
y[(2 i-1) / 2] \sim \bar{x}_{\max , i}
$$

and. in particular.

$$
y\left(\frac{1}{2}\right) \sim \bar{x}_{\max , 1}
$$

(see below), the plot of $\bar{x}_{\max , 1}$ against $\alpha / \alpha_{\mathrm{c}}$ in Figure 2 was drawn up to the gel point. To explain the approximation (A3), we remark that the degree of polymerization $y\left(\frac{1}{2}\right)$, beyond which a system on average contains $\frac{1}{2}$ of a molecule, leads to the following one-to-one correspondence: for each molecule in the ensemble of $D P>y\left(\frac{1}{2}\right)$, there is one molecule in the ensemble which is the largest in its reaction system and for which $D P<y\left(\frac{1}{2}\right)$. We show that in fact $y\left(\frac{1}{2}\right)$ is a lower limit for $\bar{x}_{\max , 1}$. The difference in size between the $n$ th-largest and $(n+1)$ th-largest molecule in a system tends statistically to increase strongly with decreasing $n$ (cf. Figure 3 ). This follows from equation (A1) (using equation 2), and at the gel point the same situation is illustrated by the proportionality to $y$ of the number-average $D P_{n}$ of the tail beyond $y$, i.e.

$$
D P_{n}(\text { of tail beyond } y)=\int_{y}^{\infty} w_{x} \mathrm{~d} x / \int_{0}^{\infty}\left(w_{x} / x\right) \mathrm{d} x=3 y
$$

(using the asymptotic distribution $w_{x}=x^{-\frac{3}{2}}$ ). Thus we see that the mean sizes $x_{\max , 1}$ of the largest molecules in those systems which have $x_{\max , 1}<$ $y\left(\frac{1}{2}\right)$ lies closer to $y\left(\frac{1}{2}\right)$ than the mean size of all molecules of size $>y\left(\frac{1}{2}\right)$. Since the two sets of molecules thus compared are in one-to-one correspondence, we have proved

$$
y\left(\frac{1}{2}\right)<\bar{x}_{\max , 1}
$$

It is also easy to show that at the gel point

$$
y\left(\frac{1}{2}\right)=2^{\frac{2}{3}} y(1)
$$

and by equation (A3) that the $D P_{n}$ of the tail beyond $y(1)$ is

$$
D P_{n}(\text { of tail beyond } y(1))=3 y(1)=3 \times 2^{-\frac{2}{3}} \times y\left(\frac{1}{2}\right) \approx 1.9 y\left(\frac{1}{2}\right)
$$

In words, this $D P_{n}$ is the mean size of molecule per system beyond that limit of size at which, on average, a system at the gel point contains just one molecule. But this quantity is easily seen to be an upper bound for $\bar{x}_{\max , 1}$ : there is now a one-to-one correspondence between the set $\mathrm{A}$ of the largest molecules of systems for which $x_{\max }<y(1)$ and the set B of molecules which are of size $>y(1)$ but are not the largest molecules in their respective systems. In forming the average $D P_{n}$ (of tail beyond $y(1)$ ), we are including the set $\mathrm{B}$, while in calculating $\bar{x}_{\max , 1}$ the set A takes its place. Because the mean of the DP of members of A lies closer to $y(1)$ than the mean of members of B, the DP(of tail beyond $y(1))$ is an upper bound for $x_{\max }$, so that altogether

$$
y\left(\frac{1}{2}\right)<\bar{x}_{\max , 1}<1.9 y\left(\frac{1}{2}\right)
$$

at the gel point. These error bounds hold a fortiori at conversions less than the gel point.

After the gel point, the gel molecule is the largest in the system by a tremendous margin; the second-largest molecule in each system is the largest 
molecule of the sol fraction (Figure 2). The sol-fraction distribution I of conversion $\alpha$ is (even in presence of FSSE) indistinguishable from a pre-gel distribution II of over-all conversion $\alpha$. Accordingly, the largest molecule follows the same statistics in both samples, I and II; hence, plots a and $\mathrm{c}$ in Figure 2 are asymptotically symmetrical near the gel point $\left(\alpha / \alpha_{c}=1\right)$.

\section{Appendix B: Limiting forms of cubic equation for the early post-gel region of the modulus}

For the reference case of random $f$-functional polycondensation, the jump in $\mathrm{d} v / \mathrm{d} \alpha$, as $\alpha$ passes through $\alpha_{\mathrm{c}}$ at the gel point, is

$$
(\mathrm{d} v / \mathrm{d} \alpha)=\left\{\begin{array}{l}
0 \text { for } \alpha=\alpha_{\mathrm{c}}^{-} \\
-2 /(f-2) \text { for } \alpha=\alpha_{c}+
\end{array}\right.
$$

The limiting form for the number of EANCs per repeat unit is found from equations (17)-(19) by a perturbation calculation:

$$
N_{\mathrm{e}}=2 \frac{f(f-1)}{(f-2)^{2}}\left(\frac{\alpha}{\alpha_{\mathrm{c}}}-1\right)^{3}\left\{1-\frac{7 f-12}{3(f-2)}\left(\frac{\alpha}{\alpha_{\mathrm{c}}}-1\right) \pm \mathrm{o}\left(\frac{\alpha}{\alpha_{\mathrm{c}}}-1\right)\right\}
$$

The corresponding treatment of equations (20)-(24) for the case of random pairwise crosslinking (i.e. vulcanization) of distributions of primary chains which are arbitrary apart from the approximation $D P_{n} \gg 1$ yields

$$
N_{\mathrm{e}}=\frac{2 D P_{n \mathrm{p}} D P_{\mathrm{wp}}}{D P_{z \mathrm{p}}^{2}}\left(\frac{\alpha}{\alpha_{\mathrm{c}}}-1\right)^{3}\left\{1-0\left(\frac{\alpha}{\alpha_{\mathrm{c}}}-1\right)\right\}
$$

where $N_{\mathrm{e}}$ is now the number of EANCs per primary chain, and the subscript $\mathrm{p}$ refers to the primary chain distribution.

For long homodisperse primary chains, the correction term has been calculated:

$$
N_{\mathrm{e}}=2\left(\frac{\alpha}{\alpha_{\mathrm{c}}}-1\right)^{3}\left\{1-\frac{22}{7}\left(\frac{\alpha}{\alpha_{\mathrm{c}}}-1\right) \pm \mathrm{o}\left(\frac{\alpha}{\alpha_{\mathrm{c}}}-1\right)\right\}
$$

Finally, the analogous perturbation of equations (27)-(29) and (31) produces the following cubic approximation for the case of junction zones formed randomly from $n$ chains (each with $x$ active sites):

$$
N_{\mathrm{e}}=\frac{2 x(x-1)}{\{(n-1)(x-1)-1\}^{2}}\left(\frac{\alpha}{\alpha_{\mathrm{c}}}-1\right)^{3}\left\{1 \pm 0\left(\frac{\alpha}{\alpha_{\mathrm{c}}}-1\right)\right\}
$$

For large $x$, this rapidly approaches the form:

$$
N_{\mathrm{e}} \sim \frac{2}{(n-1)^{2}}\left(\frac{\alpha}{\alpha_{\mathrm{c}}}-1\right)^{3}
$$

Both equations (B5) and (B6) show $N_{\mathrm{e}}$ to be sensitive to the number of primary chains entering into each junction zone. 


\section{REFERENCES}

${ }^{1}$ P. J. Flory, J. Amer. Chem. Soc. 63, 3083, 3091, 3096 (1941).

${ }^{2}$ W. H. Stockmayer, J. Chem. Phys. 11, 45 (1943).

${ }^{3}$ J. H. Gibbs and E. A. DiMarzio, J. Chem. Phys. 30, 271 (1959);

A. Silberberg and R. Simha, Biopolymers, 6, 479 (1968).

${ }^{4}$ A. Charlesby, Proc. Roy. Soc. A222, 542 (1954).

${ }^{5}$ For earlier history see, e.g., T. E. Harris, The Theory of Branching Processes. Springer: Berlin (1963).

${ }^{6}$ J. Scanlan, J. Polymer Sci. 43, 397 (1960).

${ }^{7}$ L. C. Case, J. Polymer Sci. 45, 501 (1960).

${ }^{8}$ M. Gordon and W. B. Temple, J. Chem. Soc. $(A), 729$ (1970).

${ }^{9}$ W. B. Temple, Ph.D. Thesis, University of Essex, 1970; cf. ref. 24.

${ }^{10}$ C. Peniche Covas, S. B. Dev, M. Gordon, M. Judd and K. Kajiwara, Discussion of the Faraday Division on Gels and Gelling Processes, 1974, in press.

${ }_{11}$ M. Gordon and G. R. Scantlebury, Proc. Roy. Soc. A292, 380 (1966).

12 L. C. D. Groenweghe, J. H. Payne and J. R. Van Wazer, J. Amer. Chem. Soc. 82, 5305 (1960).

${ }^{13}$ G. Pólya, Acta Math. 68, 145 (1937).

${ }^{14}$ P. Whittle, Proc. Camb. Phil. Soc. 61, 475 (1965).

15 D. Gingell, J. Theor. Biol. 38, 677 (1973).

${ }_{16}^{16}$ P. J. Flory, Discussion of the Faraday Division on Gels and Gelling Processes, 1974, in press.

${ }^{17}$ P. Whittle, Proc. Roy. Soc. A285, 501 (1965).

${ }^{18}$ G. R. Dobson and M. Gordon, J. Chem. Phys. 43, 705 (1965).

${ }^{19 a}$ M. Gordon and T. G. Parker, Proc. Roy. Soc. (Edin.) A69, 181 (1970/1).

${ }^{19 b}$ For the recent solution of the problem see: M. Gordon and C. G. Leonis, Proc. 5th Brit. Combinatorial Conference 1975, Edited by C. St-J. Nash-Williams and J. Sheehan, Utilitas Mathematica, in press.

${ }^{20}$ M. Gordon, T. C. Ward and R. S. Whitney, in A. J. Chompff and S. Newman (eds.), Polymer Networks, p 1. Plenum: New York, London (1970).

21 P. Whittle, Suppl. Advan. Appl. Prob. 199 (1972).

22 M. Gordon and G. R. Scantlebury, J. Chem. Soc. 1 (1967).

${ }^{23}$ M. Gordon and W. B. Temple, Makromol. Chem. 160, 263 (1972); W. B. Temple, Makromol. Chem. 160, 277 (1972).

${ }^{24}$ M. Gordon, J. A. Love, T. G. Parker and W. B. Temple, J. Prakt. Chem. 313, 411 (1971).

${ }^{25}$ S. B. Ross-Murphy, Ph.D. Thesis, University of Essex, 1974, and in press.

${ }^{26} \mathrm{H}$. M. James and E. Guth, J. Chem. Phys. 11, 455 (1943).

${ }^{27} \mathrm{M}$. Gordon, submitted to Macromolecules.

${ }^{28}$ S. Strella and A. A. Bibeau, J. Macromol. Chem. 1, 417 (1966).

29 M. Gordon, Trud. 1969-Meshdinarodnoi Conference Kautsh $i$ Resine, Chimia: Moscow (1971).

30 A. V. Tobolsky, D. J. Metz and R. B. Mesrobian, J. Amer. Chem. Soc. 72,1946 (1950).

31 J. P. Berry and W. F. Watson, J. Polymer Sci. 18, 201 (1955).

${ }^{32}$ L. Mullins and A. G. Thomas, J. Polymer Sci. 43, 13 (1960).

${ }^{33}$ C. Cohen, J. H. Gibbs and P. D. Fleming III, J. Chem. Phys. 59, 5511 (1973).

34 J. H. L. Henson, A. J. Lovett and G. S. Learmonth, J. Appl. Polymer Sci. 11, 2543 (1967).

${ }^{35}$ See refs. 29, 37.

${ }^{36}$ Th. G. Scholte, J. Polymer Sci., A-2, 9, 1553 (1971).

${ }^{37}$ M. Gordon, S. Kuchárik and T. C. Ward, Coll. Czech. Commun. 35, 3252 (1970).

${ }^{38} \mathrm{~K}$. Dušek. Discussion of the Faraday Division on Gels and Gelling Processes, 1974, in press.

${ }^{39} \mathrm{~K}$. Dušek, J. Phys. Chem., in press.

${ }^{40}$ M. Gordon and C. G. Leonis, J. Chem. Soc. Faraday I (two papers, in press).

${ }^{41}$ K. Kajiwara and M. Gordon, J. Chem. Phys. 59, 3623 (1973).

42 T. A. Bryce. A. A. McKinnon, E. R. Morris, D. A. Rees and D. Thom, Discussion of the Faraday Division on Gels and Gelling Processes, 1974, in press.

43 J. D. Ferry, Advan. Protein Chem. 4, 1 (1948). 